\title{
CONTRIBUIÇÃO PARA O ESTUDO DO SIGNIFICADO DA EVOLUÇÃO DO COEFICIENTE DE MORTALIDADE INFANTIL NO MUNICIPIO DE SÃO PAULO, SP (BRASIL) NAS TRES ÚLTIMAS DÉCADAS (1950-1979)
}

Carlos Augusto Monteiro*

\begin{abstract}
MONTEiRo, C.A. Contribuição para o estudo do significado da evolucão do coeficiente de mortalidade infantil no municipio de Sāo Paulo, SP (Brasil) nas três última décadas (1950-1979). Rev. Saúde públ., S. Paulo, 16:7-18, 1982.
\end{abstract}

RESUMO: Buscando conțribuir ao estudo da relação evolução da mortalidade infantil - evolução da qualidade de vida, foram examinadas no município de São Paulo as correlações existentes nas três últimas décadas entre as séries históricas da mortalidade e as séries históricas do valor do salário mínimo e da cobertura do abastecimento público de água. Estes dois últimos, saláric e água, entendidos como fatores de maior e menor abrangência para o conjunto das condições de vida da população. $O$ descenso da mortalidade na década de 50 e o ascenso da mesma na década de 60 estiveram significativamente relacionados à evolução do salário-mínimo real. Entretanto, a evolução da mortalidade na década de 70, com importante queda a partir de 1974, esteve relacionada especificamente à evolução do abastecimento de água. Conclui-se que no período 1950-1979 são diferentes as implicações para a qualidade de vida que podem ser tiradas a partir da evolução da mortalidade infantil e que parece equivocado afirmar-se que a reversão das altas mortalidades a partir de 1974 tenha significado idêntica reversão na deterioração das condições de vida que ensejaram 0 ascenso da mortalidade no periodo anterior.

UNITERMOS: Mortalidade infantil, São Paulo, SP, Brasil. Qualidade de vida. Renda, Āgua, abastecimento.

\section{INTRODUÇAO}

O coeficiente de mortalidade infantil número de óbitos de menores de um ano para cada mil nascimentos vivos - apesar de sua especificidade, freqüentemente é considerado como medida sintetizadora da qualidade de vida de uma dada população e, portanto, do seu nivel de desenvolvimento $5,1=$.

A justificativa teórica para a elevada "sensibilidade social" do coeficiente de mortalidade infantil tem repousado na inconteste e ampla influência que os principais determinantes do nivel de vida - alimen- tação, moradia, acesso a serviços de saúde, entre outros - exercem sobre a probabilidade de sobrevivência no primeiro ano. Sua comprovação empírica, por outro lado, frequientemente é buscada seja ao nivel da relação inversa que a evolução secular do coeficiente manteve com o progredir da industrialização ${ }^{5}, 16$, seja ao nivel dos estudos transversais de distribuição de taxas de mortalidade segundo classes sociais ${ }^{1}$.

A relação de oposição que a mortalidade infantil mantém com o nivel sócio-tconômico tem persistido tanto na história dos

* Do Departamento de Nutrição da Faculdade de Saúde Pública da USP - Ar. Dr. Arnaldo, 715 - 01255 - São Paulo, SP, - Brasil. 
MONTEIRO, C.A. Contribuição para o estudo do significado da evolução do coeficiente de mortalidade infantil no municipio de São Paulo, SP (Brasil) nas três últimas décadas (1950-1979). Rev. Saúde públ., S. Paulo, 16:7-18, 1982.

paises hoje denominados desenvolvidos como na história dos não-desenvolvidos ${ }^{18}$. Apesar da redução dos níveis absolutos da mortalidade e da ampliação do arsenal da tecnologia médica e sanitária, os diferenciais sócio-econômicos da mortalidade infantil continuam a existir em ambos os grupos de países. A explicação que parece mais plausível para a manutenção destas diferenças, seria a de que o acesso aos novos fatores que surgem para a redução da mortalidade deve estar se processando de modo tão seletivo quanto o acesso aos demais elementos que definem o nivel da qualidade de vida e que tem distinguido as classes sociais 1.

Quanto à relação que a evolução secular da mortalidade infantil vem mantendo com a evolução da qualidade de vida, a mesma se reveste de caráter intrinsecamente dinâmico, ao longo do tempo e das estruturas sociais. Isto em função da variação possivel na composição dos fatores que contribuem para reduzir ou mesmo aumentar a mortalidade. Nesta variação assume importância o peso relativo ocupado pelos fatores segundo sua relação com a determinação mais geral da qualidade de vida. Há que se considerar o desempenho dos fatores mais abrangentes para a qualidade de vida em geral, como o nível de renda da população e a educação, o desenvolvimento daqueles fatores de menor abrangência, como a assistência hospitalar e o abastecimento de água, e mesmo há que se levar em conta - comportamento de fatores com ligação pouco objetiva à qualidade de vida, como os padrões de fertilidade e os movimentos migratórios.

Indícios de predominio progressivo de fatores menos abrangentes em relação à qualidade de vida podem ser detectados no acompanhamento secular da evolução dos coeficientes de mortalidade dos países europeus e dos EUA. Nestes países, na segunda metade do século passado, a importante redução da mortalidade teria sido devida basicamente à melhoria nas condiçōes gerais de vida - alimentação, moradia, higiene - provenientes dos avanços obtidos na agricultura e do advento da revolução industrial 14. Já neste século, a seqüente e não menos importante redução de mortalidade teria sido devida menos à continuada melhoria nas condições gerais de vida do que ao progressivo emprego e aperfeiçoamento de específicos recursos médicos e de saúde pública ${ }^{14}$.

Outra ainda parece ter sido a composição dos fatores - e, portanto, o significado da redução dos níveis de mortalidade dos países não desenvolvidos. Não participantes da revolução industrial do século passado, estes países reduziram sensivelmente seus niveis de mortalidade apenas neste século. Estas reduções, que se processaram inclusive em um prazo de tempo relativamente curto, teriam sido creditadas, entretanto, muito mais a algumas ações de saúde pública, principalmente na forma de campanhas de saneamento impulsionadas por financiamento e tecnologia procedentes dos países desenvolvidos, do que propriamente a mudanças substanciais havidas nas condições gerais de vida de suas populações ${ }^{2,19}$. Mais recentemente, a revisão acurada dos resultados de dez projetos de extensão de cuidados de saúde por pessoal auxiliar, conduzidos nas três últimas décadas em diferentes localidades pobres do mundo, permitiu a seus autores sustentarem a hipótese de que "pacotes simplificados de saúde", inteligentemente concebidos, que privilegiem o grupo materno-infantil e as atividades de imunização e vigilância nutricional, possam por si, e em um prazo de um a cinco anos, reduzir de um terço à metade a mortalidade infantil vigente em regiões atrasadas ?.

Além das modificaçōes surgidas ao nivel do emprego de técnicas e de recursos de saúde, outro aspecto que pode alterar a composição dos fatores responsáveis pela evolução da mortalidade infantil em países desenvolvidos e não desenvolvidos são os padrões de fertilidade e de natalidade. Ao se modificarem as proporções dos nascimentos advindos de estratos de maior ou de 
MONTEIRo, C.A. Contribuiçáo para o estudo do significado da evolução do coeficiente de mortalidade infantil no município de São Paulo, SP (Brasil) nas três últimas décadas (1950-1979). Rev. Saúde públ., S. Paulo, 16:7-18, 1982.

menor risco, necessariamente se altera a taxa de mortalidade da população, ainda que as taxas de mortalidade específicas para os estratos tenham permanecido as mesmas. Nos Estados Unidos, por exemplo, a simples redução, entre 1965 e 1972, na proporção de nascimentos provenientes de mulheres em idades extremas ou com elevada paridade teria sido responsável de "per si" por $27 \%$ do decréscimo observado no período na mortalidade infantil 13. No Chile, idêntico fenômeno entre 1965 e 1975 teria sido responsável por $13 \%$ da redução da mortalidade, sendo possivelmente bem maior este percentual se além da variação da natalidade segundo características de idade e paridade, fosse considerada a variação conforme características sócio-econômicas da população ${ }^{17}$.

O estudo do significado que no município de São Paulo tem assumido a relação evolıção da mortalidade infatil - evolução da qualidade de vida se afigura promissor em função de três razōes básicas: a) a condição singular do município de ser uma localidade de um país não desenvolvido com um sistema razoável de registro de estatísticas vitais; b) o caráter hegemônico da economia de São Paulo diante do processo nacional de desenvolvimento; c) a inusitada evolução de sua mortalidade infantil em declínio na década de 50, em intensa elevação na década de 60 e novamente em declínio acentuado na década de 70 .

Estudando o período 1950-1970, Leser ${ }^{9}$ vê a evolução da mortalidade infantil em São Paulo como retrato importante da modificação do poder aquisitivo da população, decorrente da fixação do salário-minimo em niveis crescentes na década de 50 e em niveis decrescentes na década de 60 . Ainda que apenas visualizada em gráfico, chamava a atenção, no estudo de Leser ${ }^{\circ}$, a evidente relação inversa que se fazia presente no período 1950-70 entre o valor do salário-mínimo e o nivel da mortalidade infantil. Como elementos de encadeamento de seu raciocínio, Leser ${ }^{9}$ lembrava a comprovada relação em São Paulo entre nivel de renda e adequação da alimentação e entre esta e a desnutrição infantil com toda sua gama de influências sobre o obituário do primeiro ano de vida.

$\mathrm{Na}$ década de 70 , porém, sem que se detecte uma valorizaçāo do salário-mínimo de modo a repor parte substancial de seu melhor poder aquisitivo, ocorre uma brusca redução na mortalidade infantil do municipio levando-a de volta aos valores mínimos encontrados ao final da década de $\mathbf{5 0}$, quando o salário-mínimo alcançara os seus valores mais altos.

Por ser fato muito recente, a redução da mortalidade na década de 70 ainda não mereceu estudos mais aprofundados. Há especulações que vão da negação pura $e$ simples da confiabilidadie dos dados - o que, diga-se de passagem, não procede - a conclusões apressadas e absolutamente não fundamentadas dos que, fazendo uma leitura inversa da década de 60 , sustentam que as condições gerais de vida da população, de fato, teriam melhorado sobremaneira ao final dos anos 70 .

O propósito deste trabalho, como primeira tentativa de enfocar periodo tão recente, é examinar, nas três últimas décadas, ano a ano, a evolução da mortalidade infantil ao lado da evolução de potenciais determinantes da mortalidade que se caracterizem por maior ou menor abrangência em relação à determinação das condições gerais da qualidade de vida da população. Em função da disponibilidade de dados sem solução de continuidade para o periodo e da estrutura da mortalidade infantil em São Paulo, onde pontifica a doença diarréica, optou-se por eleger como fator determinante da mortalidade com ampla abrangência para o restante das condições de vida, o valor do salário-minimo, e como fator determinante de menor abrangência, a cobertura da rede pública do abastecimento de água. 
MONTEIRo, C.A. Contribuição para o estudo do significado da evolução do coeficiente de mortalidade infantil no município de São Paulo, SP (Brasil) nas três últimas décadas (1950-1979). Rev. Saúde públ., S. Paulo, 16:7-18, 1982.

\section{METODOLOGIA}

Os coeficientes de mortalidade infantil utilizados neste estudo, até 1970 , são procedentes do então Departamento de Estatística do Estado de São Paulo (Leser ${ }^{9}, 1975$ ); a partir de 1971 procedem da Central de Dados e Referências da Fundação SEADE (Sistema Estadual de Análise de Dados). Para manter a homogeneidade da série histórica foram utilizados os coeficientes segundo local de ocorrência, os únicos disponiveis para todo o periodo. Estes coeficientes se baseiam no total de nascimentos vivos e óbitos menores de um ano registrados no município de São Paulo em um determinado ano. Com isto, óbitos e nascimentos de residentes fora do município, que ali acorrem, em geral, à procura de serviços de saúde, são computados no coeficiente de São Paulo. Tal fato, por ser mais freqüente no caso de óbitos, superestima a real mortalidade do municipio. Entretanto, há eviđências de que o fenômeno de invasão de registros tem estado presente no municipio por todo 0 período ${ }^{3,4,8}$, sem modificações que possam de "per si" ter alterado de forma sensível a evolução do coeficiente. Quanto ao não registro, em São Paulo, de nascimentos e óbitos de seus residentes porque tenham ocorrido em outros municípios, ou porque não tenham sido de fato registrados, ou porque se trate de recém-nascidos vivos que morrem logo após o parto e são erroneamente classificados como natimortos - sua proporção é relativamente pequena ${ }^{8}$, não havendo igualmente, neste caso, evidências de que o fenômeno venha se alterando de forma importante no período.

O valor do salário mínimo foi calculado e expresso em termos do salário-mínimo real, ou seja do valor nominal do salário-mínimo deflacionado em relação ao custo de vida. O salário-mínime real que caracteriza cada ano do estudo é o resultado da média dos valores alcançados pelo mesmo nos doze meses do ano. Em prol de maior clareza na exposição dos resultados, os valores em cruzeiros do salário-mínimo real foram transformados em números índices com todos os valores da série referidos ao maior salário, observado em 1959, ao qual se atribuiu valor 100. Para toda a série, a fonte dos valores nominais do salário mínimo foi a Secretaria dos Negócios Metropolitanos do Estado de São Paulo e os índices do custo de vida foram os calculados pelo Departamento de Estatística da Bolsa de Mercadorias de São Paulo.

Como expressão da cobertura do abastecimento de água foi considerada a proporção estimada dos domicílios do município ligados à rede pública de água. A estimativa destas proporções, em cada ano, se baseou no número de edificações da cidade ligadas à rede de água, na projeção populacional e na estimativa do número de pessoas servidas por ligação. Até 1973 foram utilizadas as estimativas adotadas por Leser e col.10 no diagnóstico oficial das condições de saúde da região metropolitana de São Paulo. A partir de 1974, as estimativas foram feitas pelo autor deste trabalho, observando número de ligações e projeção populacional procedentes da Companhia de Saneamento Básico do Estado de São Paulo (SABESP) e adotando um número médio de 5,6 pessoas servidas por ligação. Este último número resultou da decisão de compatibilizar as duas séries de estimativas, igualando arbitrariamente a cobertura de 1974 a de 1973 , biênio onde, de fato, esteve estagnada a expansão da rede de água.

A relação que a evolução de cada uma das variảveis independentes, salário-minimo real e cobertura do abastecimento de água, manteve com a variável dependente, coeficiente de mortalidade infantil, foi apreciada, nas três décadas do estudo, pelo cálculo do coeficiente de correlação ${ }^{15}$ entre as respectivas séries históricas. Em virtude de possível correlação entre as variáveis independentes, foram também calculados, para cada uma das três décadas, os coeficientes de correlação parcial 15. Estes identificaram, 
MONTEIRO, C.A. Contribuição para o estudo do significado da evolução do coeficiente de mortalidade infantil no município de São Paulo, SP (Brasil) nas três últimas décadas (1950-1979). Rev. Saúde públ., S. Paulo, 16:7-18, 1982.

sucessivamente, a ação da evolução do salário independentemente da influência da cobertura de água e a ação da evolução da cobertura de água independentemente da influência dos salários.

O significado estatístico dos coeficientes de correlação foi examinado através do cômputo da estatística " $t$ " e da consulta à distribuição probabilística dos valores desta estatistica para oito graus de liberdade ( $n$ - 2), no caso dos coeficientes simples e para sete graus de liberdade (n - 3), no caso dos coeficientes parciais.

\section{RESULTADOS}

\section{Década de 50}

A evolução do coeficiente de mortalidade infantil e a evolução dos valores do salário-mínimo real e da cobertura do abasteci- mento de água no município de São Paulo, entre 1950 e 1959, são apresentadas na Tabela 1 e Fig. 1.

A mortalidade infantil parte de valores bastante altos em 1950-51, em torno de 90 por mil, oscila durante a primeiro metade da década e se apresenta em franco e regular descenso a partir de 1956, o que a leva à marca de 65 por mil no final da década.

O salário-mínimo real parte de valores bastante baixos em 1950-51, em torno do valor 30 , basicamente em função de que desde sua criação em 1943 não sofre nenhum reajuste; apresenta-se oscilante até 1954 e a partir daí tem tendência nitidamente crescente, chegando em 1959 ao maior valor que iria alcançar em todo o período de estudo.

T A B E L A 1

Mortalidade infantil, salário-mínimo real e cobertura do abastecimento de água no município de São Paulo, 1950-59.

\begin{tabular}{lccc}
\hline Ano & $\begin{array}{c}\text { Coeficiente de } \\
\text { Mortalidade } \\
\text { Infantil (1) }\end{array}$ & $\begin{array}{c}\text { Indice do } \\
\text { Salário-Minimo } \\
\text { Real (2) }\end{array}$ & $\begin{array}{c}\text { \% de Domicílios } \\
\text { Abastecidos (3) }\end{array}$ \\
\hline 1950 & 89,71 & 31,98 & 61 \\
1951 & 91,47 & 29,52 & 60 \\
1952 & 71,00 & 79,33 & 59 \\
1953 & 79,16 & 65,23 & 58 \\
1954 & 70,72 & 79,77 & 57 \\
1955 & 86,51 & 89,11 & 56 \\
1956 & 86,38 & 90,52 & 54 \\
1957 & 75,54 & 98,48 & 54 \\
1958 & 70,21 & 85,67 & 55 \\
1959 & 65,42 & 100,00 & 60 \\
\hline
\end{tabular}

(1) Número de óbitos de menores de um ano para cada mil nascidos vivos. Fonte: Leser 9 (1975).

(2) Indice médio do salário-mínimo deflacionado pelo indice do custo de vida (base: ano de 1959 igual a 100).

Fonte: Secretaria dos Negócios Metropolitanos do Estado de São Paulo e Departamento de Estatística da Bolsa de Mercadorias de São Paulo.

(3) Percentagem estimada de domićlios ligados à rede pública de abastecimento de água.

Fonte: Leser 10 (1975). 
MONTEIRO, C.A. Contribuição para o estudo do significado da evolução do coeficiente de mortalidade infantil no município de Săo Paulo, SP (Brasil) nas três últimas décadas (1950-1979). Rev. Saúde públ., S. Paulo, 16:7-18, 1982.

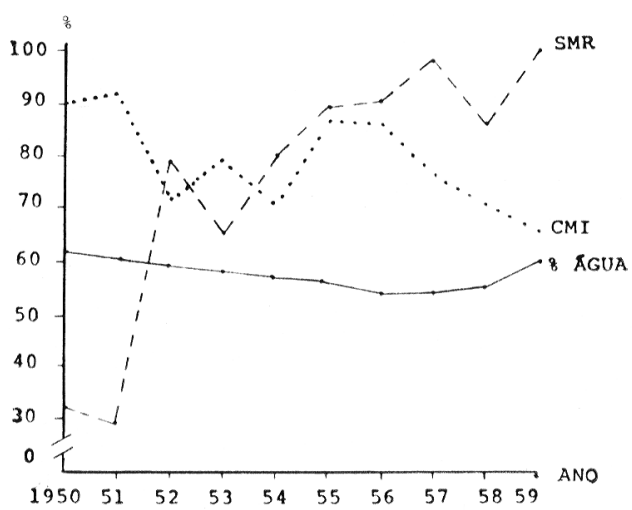

Fig. 1 - Evolução do coeficiente de mortalidade infantil (CMI), do salário-mínimo real (SMR) e da percentagem de domicílios ligados à rede pública de abastecimento de água (\% água) no municipio de São Paulo, 1950-59.

Fonte: Leser 9,10 (1975) e Departamento de Estatística da Bolsa de Mercadorias de São Paulo.

A percentagem estimada de domicílios ligados à rede pública de água, diferente da mortalidade e do salário-mínimo real, modifica-se pouco no periodo 1950-59. A cobertura é de $60 \%$ no início da década, cai lenta e regularmente nos seis primeiros anos, baixando a $54 \%$, e volta a se recuperar nos dois últimos anos da década, quando retorna aos $60 \%$.

O exame dos coeficientes de correlação verificados entre as três variáveis em estudo (Tabela 2) revela que, no periodo de 1950-59 , a evolução do coeficiente de mortalidade infantil esteve negativa e significativamente correlacionada à evolução do salário-mínimo real $(r=-0,65)$, enquanto que entre mortalidade infantil e cobertura de água não houve correlação significativa. A individualização dos efeitos do salário e da água sobre a mortalidade, obtida pela parcialização dos coeficientes de correlação, não se mostrou capaz de alterar o quadro delineado pelas correlações simples.

\section{Década de 60}

As séries históricas da mortalidade infantil, do salário-mínimo real e da cobertura do abastecimento de água no periodo 1960-69 encontram-se na Tabela 3 e Fig. 2.

$O$ coeficiente de mortalidade infantil, nos primeiros dois anos da década, mantém sua tendência à queda, chegando em 1961 a 60

T A B E L A 2

Coeficientes de correlação do salário-mínimo real (SMR) e da percentagem de domicílios lígados à rede pública do abastecimento de água (\% água) com o Coeficiente de Mortalidade Infantil (CMI), em vários periodos, no município de São Paulo.

\begin{tabular}{lcccc}
\hline & \multicolumn{2}{c}{ do SMR } & \multicolumn{2}{c}{ da \% Agua } \\
\cline { 2 - 5 } Período & $\begin{array}{c}\text { Correlação } \\
\text { simples }\end{array}$ & $\begin{array}{l}\text { Correlação } \\
\text { parcial (1) }\end{array}$ & $\begin{array}{c}\text { Correlação } \\
\text { simples }\end{array}$ & $\begin{array}{c}\text { Correlação } \\
\text { parcial (2) }\end{array}$ \\
\hline $1950-59$ & $-0,65 *$ & $-0,78 *$ & $0.11 \mathrm{Ns}$ & $-0,56 \mathrm{NS}$ \\
$1960-69$ & $-0,93 * * *$ & $-0,91 * * *$ & $-0,45 \mathrm{Ns}$ & $-0,05 \mathrm{Ns}$ \\
$1970-79$ & $-0,93 * * *$ & $-0,17 \mathrm{Ns}$ & $-0,96 * * *$ & $-0,69 *$ \\
\hline$(1)$ Correlação do SMR com o CMI quando é controlada a influência da \% água. & & \\
$(2)$ Correlação da $\%$ água com o CMI quando é controlada a influência do SMR. \\
$*$ p $<0,05$
\end{tabular}


MONTEIRo, C.A. Contribuição para o estudo do significado da evolução do coeficiente de mortalidade infantil no município de São Paulo, SP (Brasii) nas trếs últimas décadas (1950-1979). Rev. Saude públ., S. Paulo, 16:7-18, 1982.

\section{T A B E I A 3}

Mortalidade infantil, salário-minimo real e cobertura do abastecimento de água no município de São Paulo, 1960-69.

\begin{tabular}{lccc}
\hline Ano & $\begin{array}{c}\text { Coeficiente de } \\
\text { Mortalidade } \\
\text { Infantil (1) }\end{array}$ & $\begin{array}{c}\text { fndice do } \\
\text { Salário-Minimo } \\
\text { Real (2) }\end{array}$ & $\begin{array}{c}\text { \% de Domicílios } \\
\text { Abastecidos (3) }\end{array}$ \\
\hline 1960 & 62,94 & 80,46 & 60 \\
1961 & 60,21 & 90,99 & 62 \\
1962 & 64,42 & 79,29 & 64 \\
1963 & 69,90 & 73,55 & 65 \\
1964 & 67,35 & 70,49 & 65 \\
1965 & 69,38 & 69,76 & 62 \\
1966 & 73,80 & 62,59 & 61 \\
1967 & 74,31 & 60,36 & 60 \\
1968 & 76,61 & 58,98 & 60 \\
1969 & 84,34 & 57,02 & 60 \\
\hline
\end{tabular}

(1) Número de óbitos de menores de um ano para cada mil nascidos vivos. Fonte: Leser 9 (1975).

(2) Indice médio do salário-mínimo deflacionado pelo indice do custo de vida (base: ano de 1959 igual a 100).

Fonte: Secretaria dos Negócios Metropolitanos do Estado de São Paulo e Departamento de Estatística da Bolsa de Mercadorias de São Paulo.

(3) Percentagem estimada de domicílios ligados à rede pública de abastecimento de água.

Fonte: Leser 10 (1975).

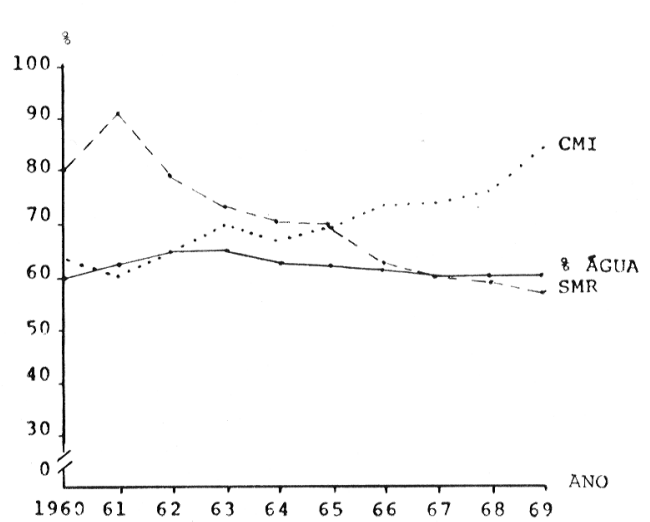

Fig. 2 - Evolução do coeficiente de mortalidade infantil (CMI), do salário-mínimo real (SMR) e da percentagem de domicilios ligados à rede pública de abastecimento de água (\% água) no município de Săo Paulo, 1960-69.

Fonte: Leser 9,10 (1975) e Departamento de Estatística da Bolsa de Mercadorias de São Paulo. por mil, o que seria o seu valor mais baixo de todo o periodo de estudo. A partir daí, e até o fim da década, apresenta tendência nitidamente crescente que o leva de volta aos niveis observados na metade dos anos 50.

O salário-mínimo real, que havia chegado a 100 em 1959, oscila nos três primeiros anos da década em torno de 80-90. A partir daí, decresce, sistematicamente, até atingir em 1969 o valor de apenas 57.

A cobertura de água continua se modificando pouco na década de 60 . Apresenta discreta elevação no início do periodo, chegando a $65 \%$ em 1963, mas decresce a partir daí, voltando no final da década aos mesmos $60 \%$ com os quais a havia iniciado. 
MONTEIRO, C.A. Contribuição para o estudo do significado da evolução do coeficiente de mortalidade infantil no município de São Paulo, SP (Brasil) nas três últimas décadas (1950-1979). Rev. Saúde públ., S. Paulo, 16:7-18, 1982.

O exame dos coeficientes de correlação verificados entre as três variáveis, no período $1960-69$, revela que novamente a evolução da mortalidade infantil esteve negativa e significantemente correlacionada à evolução dos valores do salário-mínimo real $(r=-0,93)$. Mortalidade e abastecimento de água correlacionam-se também negativamente, ainda que o coeficiente $(r=-0,45)$ não tivesse alcançado significância estatistica. A parcializaçăo dos coeficientes de correlação não modificou o quadro descrito pelas correlações simples.

\section{Década de 70}

$\mathrm{Na}$ Tabela 4 e na Fig. 3 encontram-se as evoluções da mortalidade infantil, do salário-mínimo real e da cobertura do abastecimento de água para o período 1970-79 .

O coeficiente de mortalidade infantil prossegue no início da década sua tendência de elevação, alcançando no triênio 1971-73 niveis de 93-94 por mil, que são os niveis mais altos de todos os trinta anos cobertos pelo estudo. A partir daí, e até o final da década, inicia-se um processo relativamente rápido de descenso que leva o coeficiente a 62 por mil, nível próximo das baixas mortalidades observadas no início dos anos 60 .

O salário-minimo real, que ao final da década de 60 havia sido reduzido a 57 , estaciona em torno deste valor até 1974. A partir daí, apresenta uma discreta elevação que o faz chegar em 1979 ao valor 65, ainda

T A B E L A 4

Mortalidade infantil, salário-mínimo real e cobertura do abastecimento de água no município de São Paulo, 1970-79.

\begin{tabular}{lccc}
\hline Ano & $\begin{array}{c}\text { Coeficiente de } \\
\text { Mortalidade } \\
\text { Infantil (1) }\end{array}$ & $\begin{array}{c}\text { Indice do } \\
\text { Salário-Minimo } \\
\text { Real (2) }\end{array}$ & $\begin{array}{c}\text { \% de Domićlios } \\
\text { Abastecidos (3) }\end{array}$ \\
\hline 1970 & 89,46 & 57,54 & 59 \\
1971 & 93,87 & 57,17 & 58 \\
1972 & 93,04 & 58,00 & 58 \\
1973 & 94,38 & 58,77 & 57 \\
1974 & 85,65 & 56,68 & 57 \\
1075 & 86,72 & 59,78 & 59 \\
1976 & 50,50 & 61,19 & 65 \\
1977 & 70,68 & 63,04 & 70 \\
1978 & 66,85 & 64,70 & 73 \\
1979 & 62,18 & 65,21 & 80 \\
\hline
\end{tabular}

(1) Número de óbitos de menores de um ano para cada mil nascidos vivos. Fonte: Leser 9 (1975) e Fındação SEADE.

(2) In:ice médio do salário-mínimo deflacionado pelo índice do custo de vida (base: ano de 1959 igual a 100).

Fonte: Secretaria dos Negócios Metropolitanos do Estado de Sảo Paulo e Departamento de Estatística da Bolsa de Mercadorias de São Paulo.

(3) Percentagem estimada de domicílios ligados à rede pública de abastecimento de água.

Fonte: Leser 10 (1975) e SABESP. 
MONTEIRO, C.A. Contribuição para o estudo do significado da evolução do coeficiente de mortalidade infantil no município de São Paulo, SP (Brasil) nas três últimas décadas (1950-1979). Rev. Saúde públ., S. Paulo, 16:7-18, 1982.

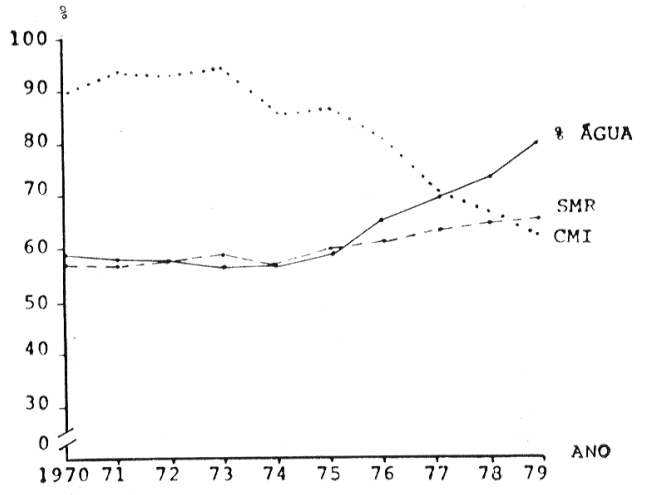

Fig. 3 - Evolução do coeficiente de mortalidade infantil (CMI), do salário-mínimo real (SMR) e da percentagem de domicílios ligados à rede pública de abastecimento de água (\% água) no município de São Paulo, 1970-79.

Fonte: Leser $\vartheta, 10$ (1975) e Departamento de Estatística da Bolsa de Mercadorias de São Paulo.

bastante distante, portanto, do valor máximo de 100 que atingira vinte anos atrás.

A cobertura do abastecimento de água na primeira metade da década, percorre caminho de lenta deterioração, reduzindo-se a $57 \%$ em 1974. A partir dai inicia uma rápida e inusitada elevação, atingindo em três e em cinco anos, sucessivamente, as marcas de 70 e $80 \%$, níveis de cobertura jamais desfrutados pela cidade em todo o período anterior de estudo.

O exame dos coeficientes de correlação verificados entre as três variáveis, no período 1970-79, revela que a evolução do coeficiente de mortalidade infantil esteve correlacionada negativa e significantemente tanto à evolução do salário-mínimo real $(\mathrm{r}=-0,93)$ quanto à evolução da cobertura de água $(\mathrm{r}=-0,96)$. A individua- lização dos efeitos exclusivos do salário-mínimo real e da cobertura de água revelou, entretanto, maior importância do abastecimento de água, cujo coeficiente de correlação parcial com a mortalidade mantém-se elevado e significativo $(r=-0,69)$, ao contrário do que ocorre com o coeficiente de correlação parcial do salário-mínimo real $(r=-0,17)$.

\section{COMENTARIOS E CONCLUSOES}

Mais do que estudo sobre a causalidade da mortalidade infantil em determinada localidade, este trabalho pretende ser uma contribuição à reflexão acerca da relação complexa que une evolução de mortalidade infantil a evolução de qualidade de vida.

Um estudo que pretendesse determinar a causalidade da mortalidade infantil, em contexto como o de São Paulo, deveria reunir informações não incluídas neste trabalho e que freqüentemente inexistem ou não são suficientemente precisas. Para o lado da mortalidade, entre outros aspectos, caberia considerar a evolução da estrutura da mortalidade infantil conforme o período do óbito, as causas de morte e os diferentes estratos sócio-econômicos da população. Para o lado dos fatores determinantes seria indispensável conhecer a evolução do poder aquisitivo real da população, cuja relação com a evolução do salário-mínimo real é apenas indicativa já que a mesma não capta, entre outros, os efeitos do desemprego, da variação no número dos que trabalham por familia e dos reajustes salariais que não acompanham o percentual de reajuste do mínimo. Obviamente também não poderia faltar a evolução da assistência de saúde e dos padrões de natalidade.

Em trabalho anterior ${ }^{11}$, referente a meados da década de 70 , constatou-se que na periferia do município de São Paulo havia elevada mortalidade infantil cujo coeficiente excedia em cerca de duas vezes o coeficiente encontrado nas áreas mais centrais da cidade. Além da maior concentração de famílias com baixa renda, notou-se que naquelas áreas periféricas da cidade encontravam-se as mais baixas coberturas de determinados serviços públicos essenciais como os centros de saúde e o abstecimento de água. Todas estas informações sugeriam que tanto alterações ao nivel da elevação global do poder aquisitivo - principalmente da população mais pobre - como a melhoria de determinados serviços públicos essenciais, poderiam 
Monteiro, C.A. Contribuiçăo para o estudo do significado da evolução do coeficiente de mortalidade infantil no município de São Paulo, SP (Brasil) nas três últimas décadas (1950-1979). Rev. Satide públ., S. Paulo, 16:7-18, 1982.

exercer grande influência sobre os niveis da mortalidade infantil do município.

Apoiado nos resultados do referido estudo e objetivando a contribuiçăo à reflexão sobre a relação mortalidade infantil qualidade de vida no contexto do município de São Paulo, procurou-se inicialmente estabelecer a evolução do coeficiente de mortalidade nas três últimas décadas. Ao lado deste, então, foram identificadas as evoluções de duas variáveis cuja ação sobre a mortalidae fosse, em ambos os casos, indiscutivel, porém cuja relação com as condições gerais de qualidade de vida da população fosse, respectivamente, de maior e de menor abrangência.

$\mathrm{Na}$ década de 50 , tem-se que a evolução da mortalidade está significativamente correlacionada à evolução do fator de maior abrangência - o valor do salário-mínimo real - levando a crer que o restante das condições de vida, do mesmo modo como a mortalidade infantil, deva ter melhorado no periodo. É inexpressiva, nesta década, a correlação entre a mortalidade infantil e o fator de menor abrangência, a cobertura do abastecimento de água, que permanece estagnada no período.

$\mathrm{Na}$ década de 60 , novamente a evolução dos valores da mortalidade se mostra significativamente correlacionada à evolução dos valores do salário-mínimo real, apenas que desta vez o periodo se caracteriza rão por sensivel melhora, mas por piora na mortalidade infantil e no salário-minimo. A correlação entre o coeficiente de mortalidade e a cobertura de água é inversa no período, idêntica à existente entre mortalidade e salário, embora não chegue a ser estatisticamente significativa, nem pareça influir na correlação alcançada entre mortalidade e salário. Pode-se supor, portanto, que na década de 60 o restante das condições de vida que dependem igualmente do poder aquisitivo da população tenham, como a mortalidade infantil, piorado no período.
$\mathrm{Na}$ década de 70 , caracterizada em sua segunda metade por acelerada redução do coeficiente de mortalidade infantil, ambas evoluções, do salário e da água, se mostraram significativamente correlacionadas à evolução da mortalidade, com coeficientes próximos a um. O estudo individualizado da ação de cada uma daquelas variáveis mostrou, entretanto, subsistência da significação da correlação apenas no caso da evolução da cobertura de água, sendo que a correlação no periodo entre mortalidade e salário parece devida na sua maior parte a uma determinada identificação da evolução dos salários com a evolução da cobertura de água. Diferentemente dos períodos anteriores, não se poderia dizer, portanto, que a evolução das demais condições da qualidade de vida da população tenha acompanhado a tendência da mortalidade, já que desta vez um fator de maior especificidade é que teria atuado sobre o coeficiente.

Diante dos resultados observados nas três décadas enfocadas pelo estudo, decorrem as conclusões seguintes:

- Em São Paulo, como em outras localidades, a interpretação da relação mortalidade infantil - qualidade de vida como uma relação simplesmente linear e de natureza inversa é extremamente perigosa, quando não, contraproducente.

- Recursos de saúde pública, que no passado exerceram importante papel na redução da mortalidade como as companhas de erradicação de vetores e outras campanhas sanitárias, ainda tem um potencial muito expressivo para a diminuição dos niveis de mortalidade infantil de localidades como São Paulo, desde o momento que sejam desenvolvidos de forma a se contraporem especificamente aos fatores de maior peso na mortalidade.

- A concomitância da implementação de recursos mais específicos de combate a mortalidade infantil, como no caso de 
MONTEIRo, C.A. Contribuição para o estudo do significalo da evolução do coeficiente de mortalidade infantil no município de São Paulo, SP (Brasil) nas três últimas décadas (1950-1979). Rev. Saúde públ., S. Paulo, 16:7.18, 1982.

São Paulo o abastecimento de água, com a melhoria das condições gerais de vida proporcionada por um poder aquisitivo crescente da população, é a situação que reúne o maior potencial para a redução do coeficiente de mortalidade infantil, e, em São Paulo, pode-se perfeitamente supor, com grande probalidade de acerto, que o encontro do salário-mínimo de $1959 \mathrm{com}$ a cobertura do abastecimento de água de 1979, que nunca existiu na prática, teria sido capaz de propiciar condiçóes para que a mortalidade infantil do municipio nunca tivesse interrompido sua tendência secular de queda e atingisse niveis realmente compativeis com a magnitude do seu desenvolvimento econômico.

\section{AGRADECIMENTOS}

Ao economista José Luiz Teixeira Vieira Marques, autor dos cálculos da série histórica do salário-minimo real; ao engenheiro Tobias Jerosolinski que nos cedeu os dados brutos do abastecimento de água a partir de 1974 e nos orientou sua interpretação; à Dra. Maria Helena D'Aquino Benício e ao Dr. Jair Lício Ferreira dos Santos pelas profícuas discussões acerca dos aspectos metodológicos e interpretativos do trabalho.

MONTEIRo, C.A. [A contribution to the study of the significance of the trends in infant mortality rates for the city of $\mathbf{S}$. Paulo, Brazil, during the last three decades (1950-1979)]. Rev. Saúde públ., S. Paulo, 16:7-18, 1982.

ABSTRACT: The possible correlations between infant mortality statistics and those, first, on the real value of the legal minimum salary and, sencondly, on the extent of the public water-supply system, for the city of $\mathbf{S}$. Paulo (Brazil) over the last three decades, were studied with a view to determining the relationship between the historical trends in the infant mortality rates and in the quality of life. The abovementioned factors - salary and water-supply - are taken as factors of greater and lesser relevance, respectively, for the overall picture of the living conditions of the population. The mortality decline in the $50 \mathrm{~s}$ and its increase in the $60 \mathrm{~s}$ were fourd to be related, significantly, to the trends in the real value of the legal minimum salary. However, the trend in mortality in the $70 \mathrm{~s}$, with a notable fall as from 1974, was found to be related specifically to the trends in water-supply extension. One may conclude that in the period $1950-1979$ the implications, relating to the quality of life, to be drawn from infant mortality trends are diverse. It would seem to be mistaken to affirm that the reversal of the high mortality rate as from 1974 might signify an identical reversal of the deterioration of living conditions which led to the increase of mortality in the preceding period.

UNITERMS: Infant mortality, S. Paulo, SP, Brazil. Quality of life. Income. Water Supply.

\section{REFERENCIAS BIBLIOGRAFICAS}

1. ANTONOVSkI, A. \& BERNSTEIN, J. Social class and infant mortality. Soc. Sci. Med., 11:453-70, 1977.

2. ARRIAGA, E. Mortality decline and its demographic effects in Latin America.
Berkeley, Institute of International Studies, University of California, 1967.

3. BARBOSA, V. Estudo comparativo entre os óbitos "de fato" $e$ "de direito" no municipio de São Paulo. São Paulo, 1967. [Tese 
MONTEIRo, C.A. Contribuição para o estudo do significallo da evolução do coeficiente de mortalidade infantil no município de São Paulo, SP (Brasil) nas três últimas décadas (1950-1979). Rev. Saúde públ., S. Paulo, 16:7-18, 1982.

de Livre-Docência - Faculdade de Saúde Pública da [SP].

4. BERQUÓ, E.S. \& GONÇALVES, M.A. A invasão de óbitos no municipio de são $P$ culo. São Paulo, Centro Brasileiro de Análise e Planejamento, 1974. (Cadernos CEBRAP, 19).

5. CHASE, H.C. Ranking countries by infant mortality rates. $P u b l$. Hlth Rep., 84:19-27, 1969.

6. GOLDSMITH, S.B. The status of health status indicators. Hlth Serv. Rep., $87: 212-20,1972$.

7. GWATKIN, D.R. et al. Can intervention make a difference? The policy implications of field experiment experience; Report to the Wold Bank. Washington. D.C., Overseas Development Council. 1979.

8. LAURENTI, R. Alguns aspectos particulares referentes aos resultados de Investigação Interamericana de Mortalidade na Infância no projeto de São Pa: lo, Brasil. Bol. Ofic. sanit. panamer., 89:1-14, 1975.

9. LESER, W. Crescimento da população da cidade de São Paulo, entre 1950 e 1970. e seu reflexo nas condições de saúde pública. Cienc. Cult., 27:244-56, 1975.

10. LESER, W.S.P. et al. Regiāo Metropolitana de São Paulo: diagnóstico 75 ; Desenvolvimento Sócio-Econômico: Saúde. São Paulo, 1975.

11. MONTEIRO, C.A. et al. Mortaliciade no primeiro ano de rida e distribuição de renda e de recursos públicos de saúde. Rer. Saúde públ., S. Paulo, 14:515-39, 1980.
12. MORIYAMA, I.M. Problems in the measurement of health status. In: Sheldon, E. \& Moore. W. Indicators of social change. New York, Ruissel Sage Fountation, 1968.

13. MORRIS, W.M. et al, Shifting age - parity distribution of births and the decrease of infant mortality. Amer. J. publ. Hith, $65: 359-62,1975$.

14. PReston, S.H. Mortality trends. Ann Rer. Soc, 3:163-78, 1977.

15. STEEL, R.G.D. \& TORRIE, J.H. Principles and procedures of statistics with special reference to the biological sciences. New York, McGraw-Hill, 1960.

16. STOCKWELL, E.G. Infant mortality and socieconomic status: a changing relationship. Milbank mem. Fd Quart. $11: 101-11,1962$.

17. TAUCHER, E. Mortalidad infantil en Chile: tendencias, diferenciales y causas, Santiago, Centro Latinoamericano de Demografia, 1979.

18. VALLIN, J. \& BEHM, H. Mortality differentials among human groups. [Paper presented to the Seminar on Biological and Social Aspects of Mortality and the Lenght of Life. International thion for the Scientific Study of Population. Fiuggi Terme, Italy, 1980].

19. WORLD HEALTH ORGANIZATION. The second decade of public health in Africa; part II. Geneva, 1968.

Recebido para publicação em 18/05/1981

Aprovado para publicaşão em 07/10/1981 